\title{
Official ERS technical standard: Global Lung Function Initiative reference values for static lung volumes in individuals of European ancestry
}

\author{
Graham L. Hall ${ }^{1,2}$, Nicole Filipow ${ }^{3}$, Gregg Ruppel $\mathbb{1}^{4}$, Tolu Okitika, \\ Bruce Thompson ${ }^{5}$, Jane Kirkby ${ }^{6}$, Irene Steenbruggen (1) ${ }^{7}$, Brendan G. Cooper ${ }^{8}$, \\ Sanja Stanojevic ${ }^{3}$, on behalf of the contributing GLI Network members ${ }^{9}$
}

@ERSpublications

The GLI Network has developed all-ages reference equations for lung volumes for populations of European ancestry. The unification of GLI lung function reference equations will improve the interpretation of lung function in patients with lung disease. https://bit.ly/3hHZR1N

Cite this article as: Hall GL, Filipow N, Ruppel G, et al. Official ERS technical standard: Global Lung Function Initiative reference values for static lung volumes in individuals of European ancestry. Eur Respir J 2021; 57: 2000289 [https://doi.org/10.1183/13993003.00289-2020].

\begin{abstract}
Background: Measurement of lung volumes across the life course is critical to the diagnosis and management of lung disease. The aim of the study was to use the Global Lung Function Initiative methodology to develop all-age multi-ethnic reference equations for lung volume indices determined using body plethysmography and gas dilution techniques.

Methods: Static lung volume data from body plethysmography and gas dilution techniques from individual, healthy participants were collated. Reference equations were derived using the LMS (lambdamu-sigma) method and the generalised additive models of location shape and scale programme in $\mathrm{R}$. The impact of measurement technique, equipment type and being overweight or obese on the derived lung volume reference ranges was assessed.

Results: Data from 17 centres were submitted and reference equations were derived from 7190 observations from participants of European ancestry between the ages of 5 and 80 years. Data from nonEuropean ancestry populations were insufficient to develop multi-ethnic equations. Measurements of functional residual capacity (FRC) collected using plethysmography and dilution techniques showed physiologically insignificant differences and were combined. Sex-specific reference equations including height and age were developed for total lung capacity (TLC), FRC, residual volume (RV), inspiratory capacity, vital capacity, expiratory reserve volume and RV/TLC. The derived equations were similar to previously published equations for FRC and TLC, with closer agreement during childhood and adolescence than in adulthood.

Conclusions: Global Lung Function Initiative reference equations for lung volumes provide a generalisable standard for reporting and interpretation of lung volumes measurements in individuals of European ancestry.
\end{abstract}

\footnotetext{
This article has supplementary material available from erj.ersjournals.com

The European Respiratory Society Global Lung Function Initiative lung volume reference equations were endorsed by the ERS Executive Committee on 2 September 2020, and have also been endorsed by the following respiratory societies: American Thoracic Society (ATS), Australian and New Zealand Society of Respiratory Science (ANZSRS), Thoracic Society of Australia and New Zealand (TSANZ), Pan African Thoracic Society (PATS) and Latin American Thoracic Association (ALAT)
}

Received: 10 July 2019 | Accepted: 27 July 2020

Copyright @ERS 2021. 


\section{Introduction}

All-age Global Lung Function Initiative (GLI) reference equations are available for spirometry [1] and transfer factor of the lung for carbon monoxide $\left(T_{\mathrm{LCO}}\right)$ [2]. Through collation of normative data from around the world, the GLI Network has developed reference equations that facilitate standardised interpretation of lung function results and improve our understanding of normal lung growth and development [3-6].

Static lung volumes (functional residual capacity (FRC), total lung capacity (TLC), residual volume (RV)) are often considered components of a "complete" pulmonary function test. Gas dilution techniques or plethysmography are required to measure FRC. The use of linked spirometry manoeuvres allows the measurement of vital capacity (VC), expiratory reserve volume (ERV) and inspiratory capacity (IC) and in combination with FRC allow the calculation of TLC and RV. Static lung volume measurements do not reveal a specific diagnosis, but rather provide a physiological pattern which can be used in conjunction with spirometry and $T_{\text {LCO }}$ tests to refine a differential diagnosis [7].

It is well accepted that individual lung volumes measured by plethysmography and gas dilution technique are not interchangeable [8]. Specifically, the difference in FRC measured by plethysmography and gas dilution in patients with obstructive lung disease is itself a measure of gas trapping. In addition, the specific properties of different dilution gases, and the techniques used to measure and calculate different indices can produce different results within an individual. However, the extent to which variations in the lung volumes acquired from different measurement techniques affects population variability and hence the upper and lower limits of normal in large, representative datasets of healthy individuals has yet to be evaluated.

Similar to other pulmonary function tests, measured lung volume values need to be compared to a reference population for appropriate interpretation [7]. Reference equations for lung volumes including the upper and lower limits of normal have been reported in children and adults [9-18]; none have spanned the full paediatric and adult age range, and none evaluate different lung volume measurement techniques. There are currently no static lung volumes reference equations recommended by any of the respiratory societies, which has resulted in inconsistent reporting and interpretation of lung volume measurements across the world.

The objective of this study was to collate lung volume data from healthy individuals and derive GLI reference ranges for static lung volume indices.

\section{Methods}

An application was approved for a European Respiratory Society (ERS) task force to develop global lung volume reference values. Task force co-chairs and members were approved by the ERS. Task force members were scientists and healthcare professionals with significant experience in international guidelines and lung function testing and knowledge of respiratory physiology and statistical modelling. Potential conflicts of interest were disclosed to and vetted by the ERS.

\section{Data sources}

Authors of published studies that included lung volume measurements in healthy individuals were contacted and invited to share their data with the GLI lung volumes task force. Information about the task force was circulated through international and local respiratory societies to solicit unpublished data or published data that had not been identified. Data were accepted from lung volume measurements made using body plethysmography as well as gas dilution techniques (e.g. helium dilution and multiple-breath washout). Details about the equipment and methodology used, as well as information relating to adherence with current international technical standards [8] were collected from the published papers,

Affiliations: ${ }^{1}$ Children's Lung Health, Wal-yan Respiratory Research Centre, Telethon Kids Institute, Perth, Australia. ${ }^{2}$ School of Physiotherapy and Exercise Science, Curtin University, Perth, Australia. ${ }^{3}$ Translational Medicine, Hospital for Sick Children, Toronto, ON, Canada. ${ }^{4}$ Pulmonary, Critical Care and Sleep Medicine, Saint Louis University School of Medicine, St Louis, MO, USA. ${ }^{5}$ School of Health Sciences, Swinburne University of Technology, Melbourne, Australia. ${ }^{6}$ Respiratory Medicine, Sheffield Children's Hospital NHS Foundation Trust, Sheffield, UK. ${ }^{7}$ Pulmonary Laboratory, Isala Klinieken, Zwolle, The Netherlands. ${ }^{8}$ Lung Function and Sleep, University Hospital Birmingham and Institute of Clinical Sciences, University of Birmingham, Birmingham, UK. ${ }^{9}$ Contributing GLI Network members included representatives of centres that submitted data and members of the GLI Network Executive; a list of the Network members can be found in the acknowledgements section.

Correspondence: Graham L. Hall, Children's Lung Health, Telethon Kids Institute, PO Box 855, West Perth, 6872, WA, Australia. E-mail: graham.hallatelethonkids.org.au 
from the authors or manufacturers directly, to confirm that methods were compatible with those currently available. As the GLI analytical team did not have access to raw data sources to confirm data quality, it was assumed that if contributors indicated that American Thoracic Society (ATS)/ERS standards were applied, then the submitted data met all acceptability and repeatability criteria of the relevant standards. All contributing authors provided explicit permission for data to be shared with the GLI Network and confirmed local regulatory approval for the collection and sharing of data. An online, secure data portal was used to capture data [19]. All data were anonymised and submitted using a standard data template; initial data queries were performed, and contributors were contacted to clarify outliers.

Health was defined as never-smoked with no history of self-reported or doctor-diagnosed respiratory disease. Outliers were identified using a priori criteria: forced expiratory volume in $1 \mathrm{~s}\left(\mathrm{FEV}_{1}\right) \mathrm{z}$-scores $>5$ or $<-5$, and height $\mathrm{z}$-scores $>5$ or $<-5$ in children (age $\leqslant 18$ years). These limits were used to identify data discrepancies and exclude participants in the extremes of the healthy population.

\section{Statistical analyses}

The generalised additive models of location shape and scale (GAMLSS) [20] modelling approach was applied [21, 22]. Briefly, the lambda-mu-sigma (LMS) method is an extension of regression analysis which includes three components: 1) the skewness (lambda), which models the departure of the variables from normality using a Box-Cox transformation; 2) the median (mu); and 3) the coefficient of variation (sigma), which models the spread of values around the median and adjusts for any non-uniform dispersion [23]. The three quantities (LMS) are allowed to change with height and/or age, to reflect changes in lung function distributions as people grow and age. We applied the LMS method using the GAMLSS package in the statistical programme R. Goodness of fit was assessed by Schwartz Bayesian criteria, Q-Q plots and worm plots.

Lung volume technique (e.g. plethysmography compared with gas dilution), equipment and centre differences were systematically compared using two approaches. Firstly, reference equations were derived for all valid data and individual z-scores were calculated for each observation. A one-way ANOVA test was used to determine whether there were any statistically significant differences between calculated z-scores for each of the lung volume methods and equipment. In each case the largest group was used as the reference and mean \pm SD of the offset relative to the reference are reported. Secondly, technique, equipment and centre were each added to the final prediction model as covariates to determine whether 1) the addition of these variables improved model fit and 2) the variables were statistically significant and independent predictors of the relevant lung volume index.

As obesity is known to affect lung volumes, the impact of being overweight or obese on lung volume indices was assessed. Normal weight included individuals whose body mass index (BMI) was $<85$ th centile for children [24], and $<25 \mathrm{~kg} \cdot \mathrm{m}^{-2}$ from adults aged $\geqslant 19$ years. JONES and NzEKwU [25] suggest that changes in FRC (and ERV) are exponential for BMI $25-30 \mathrm{~kg} \cdot \mathrm{m}^{-2}$, suggesting that a cut-off for normal weight of $25 \mathrm{~kg} \cdot \mathrm{m}^{-2}$ is appropriate. $\mathrm{z}$-scores in overweight individuals were summarised compared with normal-weight individuals.

As the majority of the data were from one centre, we examined any potential bias that might have arisen. A random sample of data were selected from this centre and reference equations were created using only the smaller dataset.

\section{Results}

\section{Study population}

Data from 17 centres in 11 countries (table 1) were submitted, giving a total of 7721 measurements to the GLI lung volume task force. Similar to previous GLI task forces, many centres contributed smaller datasets, with a smaller number of centres contributing larger datasets; one centre contributed data from 3705 individuals. The vast majority of data (97.2\%) came from participants of European ancestry. European ancestry was defined using the same classification as in the GLI spirometry analysis [1]. Data from participants of non-European ancestry were excluded (206 measurements). If FRC data were not submitted, we did not report other lung volume indices (for example VC derived from a slow VC manoeuvre). The available data, after exclusions, were from individuals aged 3.2-91.0 years and are summarised in table 2 and supplementary figure S1. Due to the small number of participants aged $<5$ years $(n=35)$, and $>80$ years $(n=79)$, the equations and look-up tables are limited to $5-80$ years. The average height of adult males was $171 \pm 7.6 \mathrm{~cm}$ (range $145-203 \mathrm{~cm}$ ), and $157.7 \pm 7.0 \mathrm{~cm}$ in females (range $134-186 \mathrm{~cm})$. In a subset of the submitted data $(\mathrm{n}=5605), \mathrm{FEV}_{1}$ and forced vital capacity (FVC) data acquired from spirometry were submitted. In these participants, predicted lung function (z-scores) was derived from the 2012 GLI spirometry equations, resulting in a mean \pm SD $z$-score for $\mathrm{FEV}_{1}$, FVC and $\mathrm{FEV}_{1} / \mathrm{FVC}$ ratio of $0.19 \pm 1.00$ (figure $1 \mathrm{a}$ ), $0.31 \pm 1.00$ (figure $1 \mathrm{~b}$ ) and $-0.23 \pm 0.83$, respectively. 
TABLE 1 Summary of data by centre before exclusions

\begin{tabular}{lccccc} 
& Participants n & Equipment & European ancestry & Obese & FEV $_{\mathbf{1}} \mathbf{z}$-score \\
\hline France & 184 & Jaeger MasterScreen & 97.8 & 10.9 & $0.24 \pm 0.92$ \\
Italy & 3705 & SensorMedics Vmax & 100.0 & 24.0 & $0.31 \pm 0.96$ \\
Australia & 128 & Eco Medics Exhalyzer & 93.0 & 8.6 & \\
Australia & 66 & SensorMedics Vmax & 100.0 & 4.6 & $0.26 \pm 0.91$ \\
French Polynesia & 113 & CosMed & 22.1 & 39.8 & $0.67 \pm 1.00$ \\
Australia & 123 & SensorMedics Spectra & 100.0 & 8.1 & $0.23 \pm 0.90$ \\
Germany & 58 & ndd EasyOne Pro Lab & 100.0 & 8.6 & \\
Tunisia & 615 & Other & 100.0 & 22.8 & $-0.31 \pm 1.16$ \\
Australia & 91 & SensorMedics Spectra & 100.0 & 4.4 & \\
Mexico & 132 & Jaeger Master Screen & 100.0 & 7.6 & $0.20 \pm 0.97$ \\
Spain & 545 & Jaeger Master Screen & 100.0 & 0.0 & $-0.02 \pm 0.90$ \\
Mexico & 164 & ndd EasyOne Pro Lab & 100.0 & 11.6 & $0.10 \pm 1.18$ \\
Mexico & 453 & ndd EasyOne Pro Lab & 100.0 & 4.4 & \\
Switzerland & 82 & Eco Medics Exhalyzer & 100.0 & 2.4 & \\
Netherlands & 723 & Jaeger Master Screen & 100.0 & 4.0 & \\
United States & 59 & Medisoft BodyBox & 0.0 & 11.9 & $0.69 \pm 0.97$ \\
United Kingdom & 160 & Jaeger Master Screen & 71.9 & 8.1 & $-0.06 \pm 1.05$
\end{tabular}

Data are presented as $\%$ or mean $\pm \mathrm{SD}$, unless otherwise stated. Summary of data submitted to the Global Lung Function Initiative (GLI) lung volumes task force. Obesity was defined as body mass index (BMI) $>95$ th centile in children and $\mathrm{BMI}>30 \mathrm{~kg} \cdot \mathrm{m}^{-2}$ in adults (aged $>18$ years). Some centres ( $\mathrm{n}=11$ ) submitted spirometry data from which the mean $\pm S D$ of the forced expiratory volume in $1 \mathrm{~s}\left(\mathrm{FEV}_{1}\right)$ z-score was calculated using the GLI 2012 spirometry equations [1].

\section{Reference values}

Sex-specific reference equations for FRC were derived (table 3, figure 2). Initial analyses of the FRC values showed significant overlap between plethysmography and gas dilution techniques across all ages (supplementary figure S2a and table S1); therefore, preliminary models combined data from all techniques.

TABLE 2 Summary of the demographic characteristics of the available data for each lung volume index

\begin{tabular}{|c|c|c|c|}
\hline & Participants & Age years & Female \\
\hline \multicolumn{4}{|l|}{ FRC } \\
\hline $\mathrm{He}$ & 683 & $22.3-87.0$ & $399(58.4)$ \\
\hline $\mathrm{N}_{2}$ & 489 & $3.2-50.0$ & 263 (53.8) \\
\hline Plethysmography & 6018 & $6.0-91.0$ & $3434(57.1)$ \\
\hline Combined techniques & 7190 & $3.2-91.0$ & $4096(57.0)$ \\
\hline \multicolumn{4}{|l|}{ TLC } \\
\hline $\mathrm{He}$ & 681 & $22.3-87.0$ & $398(58.4)$ \\
\hline $\mathrm{N}_{2}$ & 229 & $4.0-12.9$ & 113 (49.3) \\
\hline Plethysmography & 5905 & $6.0-91.0$ & $3367(57.0)$ \\
\hline Combined techniques & 6815 & $4.0-91.0$ & $3878(56.9)$ \\
\hline \multicolumn{4}{|l|}{ RV } \\
\hline Combined techniques & 5660 & $4.9-91.0$ & $3296(58.2)$ \\
\hline \multicolumn{4}{|l|}{ ERV } \\
\hline Combined techniques & 4741 & $7.1-91.0$ & $2768(58.4)$ \\
\hline \multicolumn{4}{|l|}{ IC } \\
\hline Combined techniques & 5461 & $4.0-91.0$ & $3167(58.0)$ \\
\hline \multicolumn{4}{|l|}{ vc } \\
\hline Combined techniques & 5654 & $4.9-91.0$ & $3300(58.4)$ \\
\hline \multicolumn{4}{|c|}{$\begin{array}{l}\text { Data are presented as } \mathrm{n} \text {, range or } \mathrm{n}(\%) \text {. Demographics of submitted data are presented in relation to the } \\
\text { method used to collect data leither helium (He) or nitrogen }\left(\mathrm{N}_{2}\right) \text { multiple-breath washout or } \\
\text { plethysmography). FRC: functional residual capacity; TLC: total lung capacity; RV: residual volume; ERV: } \\
\text { expiratory reserve volume; IC: inspiratory capacity; VC: vital capacity. RV was calculated as TLC - VC if VC } \\
\text { was available and as FRC - ERV if ERV was available. }\end{array}$} \\
\hline
\end{tabular}


a)

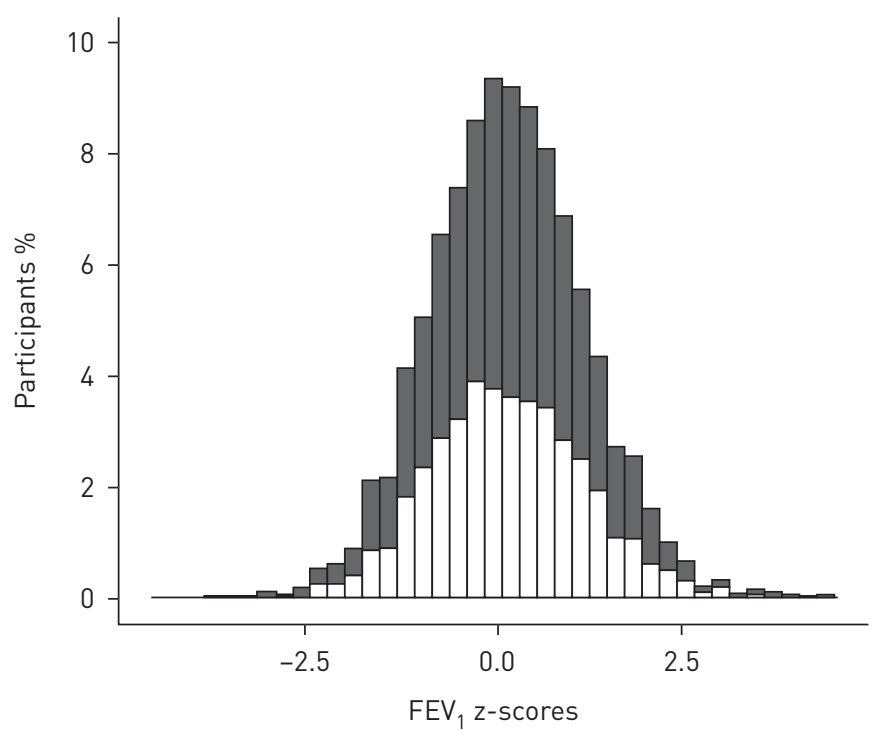

b)

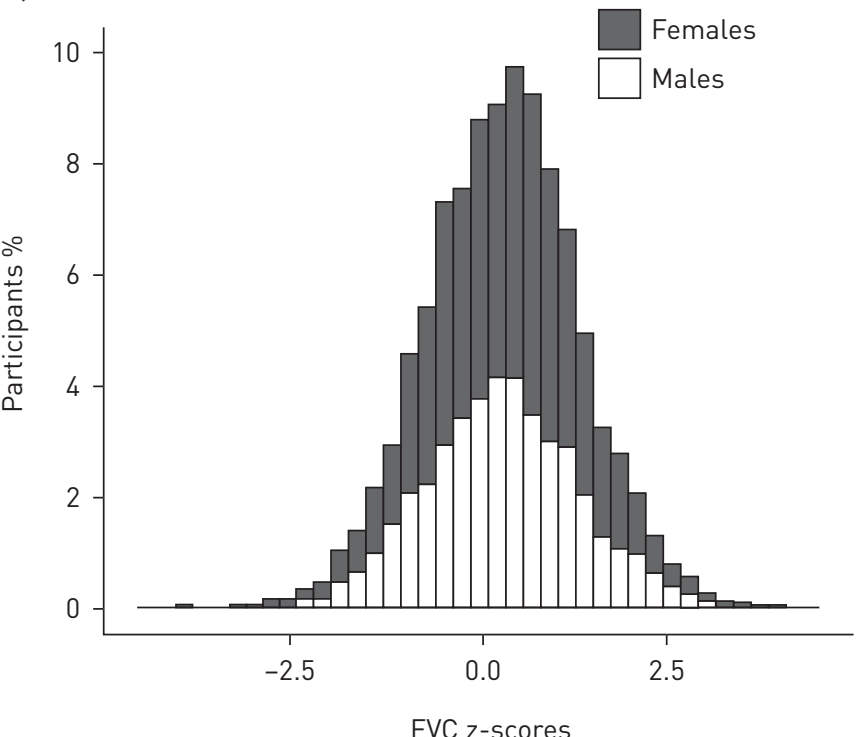

FIGURE 1 Available a) forced expiratory volume in $1 \mathrm{~s}\left(\mathrm{FEV}_{1}\right)$ z-scores and b) forced vital capacity (FVC) z-scores from participants with lung volume data. FEV 1 and FVC data were submitted from 5605 individuals with matching lung volumes data. Data were a good fit to the Global Lung Function Initiative 2012 spirometry reference equations [1] with a mean \pm SD of $0.19 \pm 1.00$ for $F E V_{1}$ and $0.31 \pm 1.00$ for FVC.

Subsequently, we tested whether the predicted values derived from the combined data systematically differed according to which method was used to measure FRC. While there were statistically significant differences between collection methods (supplementary figure S3a) and equipment type (supplementary figure S4a), these differences were not clinically or physiologically meaningful with relative differences of $<150 \mathrm{~mL}$ (supplementary table S1) and within $0.5 \mathrm{z}$-scores (supplementary figure S3a). A worked example to calculate the predicted value, limits of normal and z-scores is included in the supplementary material.

Sex-specific predicted values for TLC were derived from the combined gas dilution and plethysmographic measurements (table 3, supplementary figures S2b and S3b). Similar to the FRC values, there were statistically significant, but not clinically relevant differences in TLC between the different techniques (supplementary figure S3) or equipment (supplementary figure S4) with differences being $<200 \mathrm{~mL}$ (supplementary table S1).

TABLE 3 Equations for predicted values for the median (M), the variability around the median (S) and the skewness (L) for each of the test indices are presented for all data. Mspline and Sspline correspond to the age-varying coefficients from the look-up tables available in the supplementary material or for download from the Global Lung Function Initiative website: www.lungfunction.org

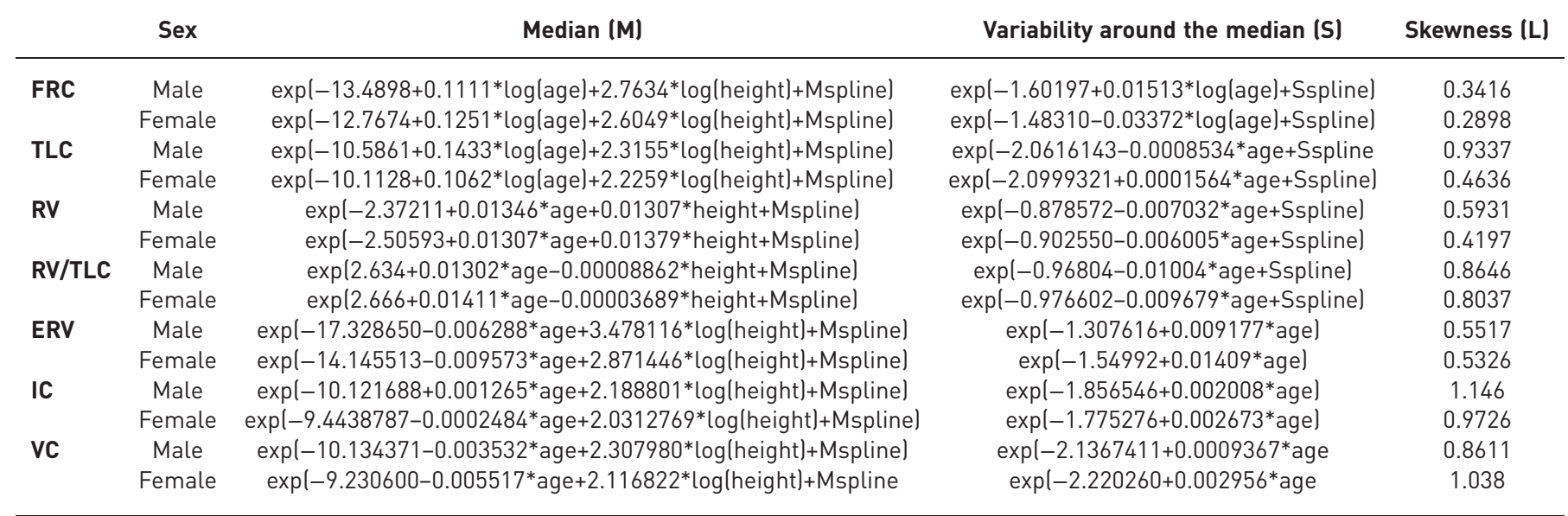

FRC: functional residual capacity; TLC: total lung capacity; RV: residual volume; ERV: expiratory reserve volume; IC: inspiratory capacity; VC: vital capacity. 
a)
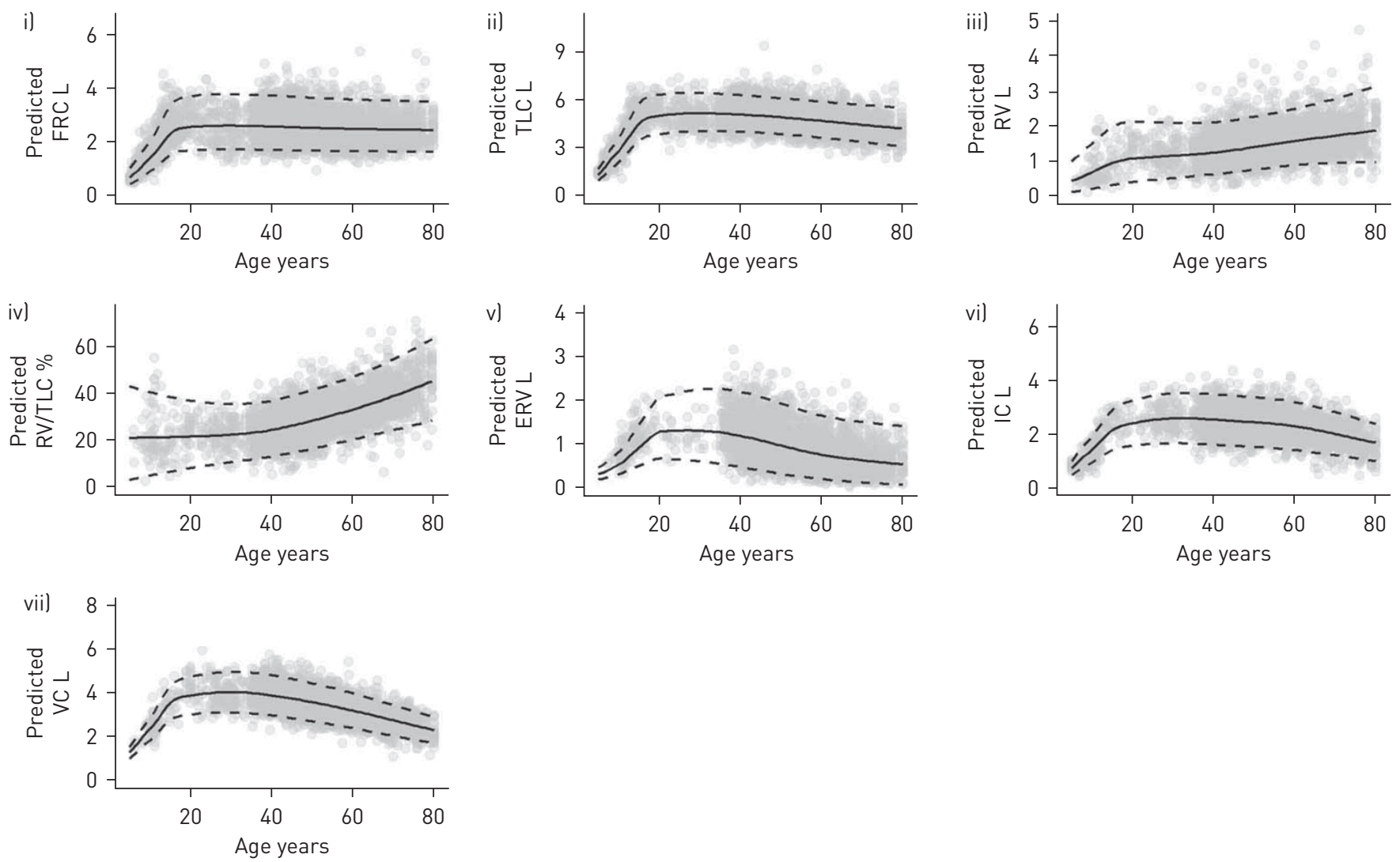

b)
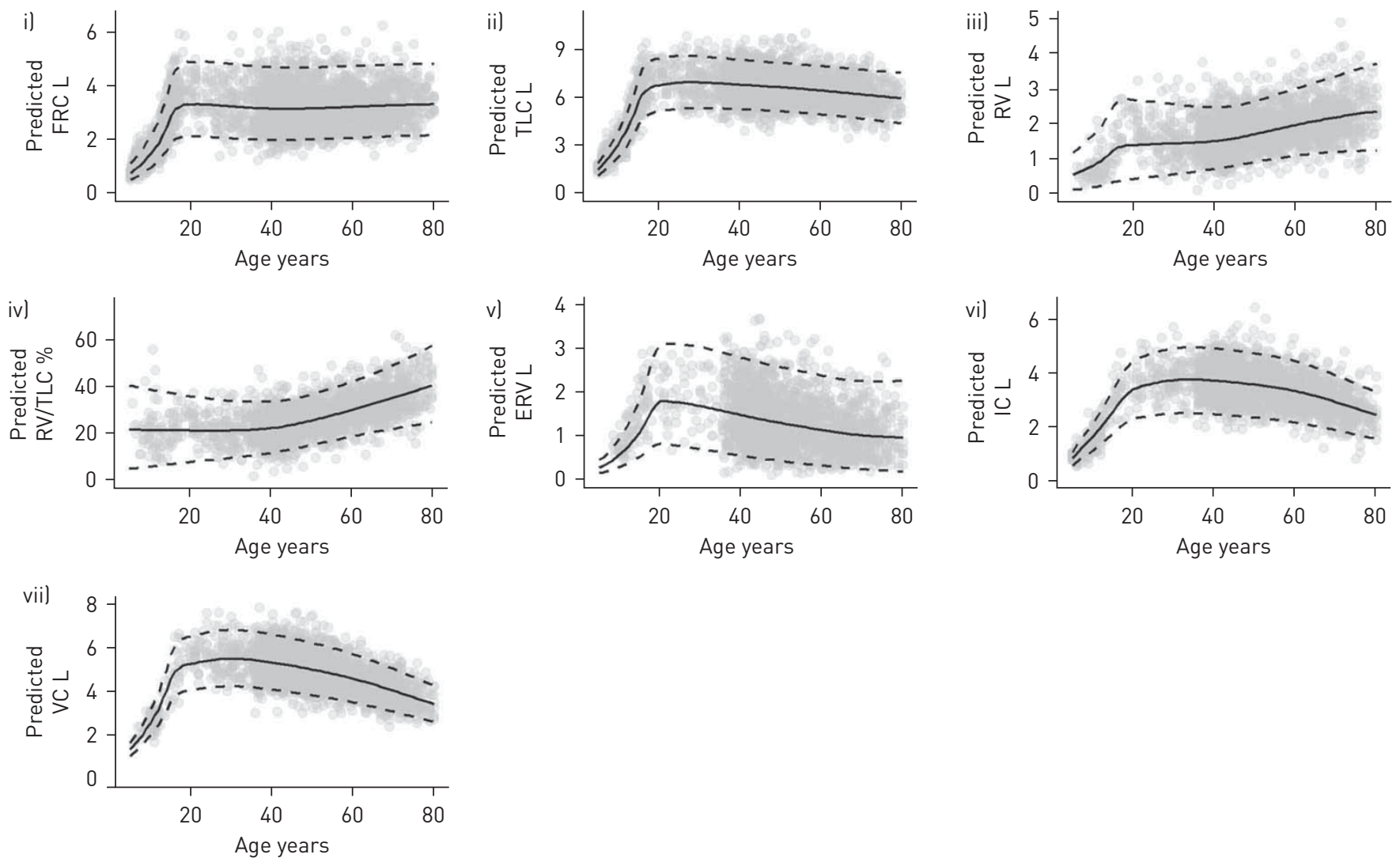

FIGURE 2 Predicted equations for a) females and b) males: il functional residual capacity (FRC), ii) total lung capacity (TLC), iii) residual volume (RV), iv) RV/TLC, v) expiratory reserve volume (ERV), vi) inspiratory capacity (IC) and vii) vital capacity (VC) across age (limits of normal). 
FIGURE 3 Between-subject variability (coefficient of variation) for functional residual capacity (FRC) and total lung capacity (TLC) for males (M) and females (F). The average coefficient of variation at each age is presented assuming the average height for individuals at each age. Coefficients of variation for other indices are included in supplementary figure S9.

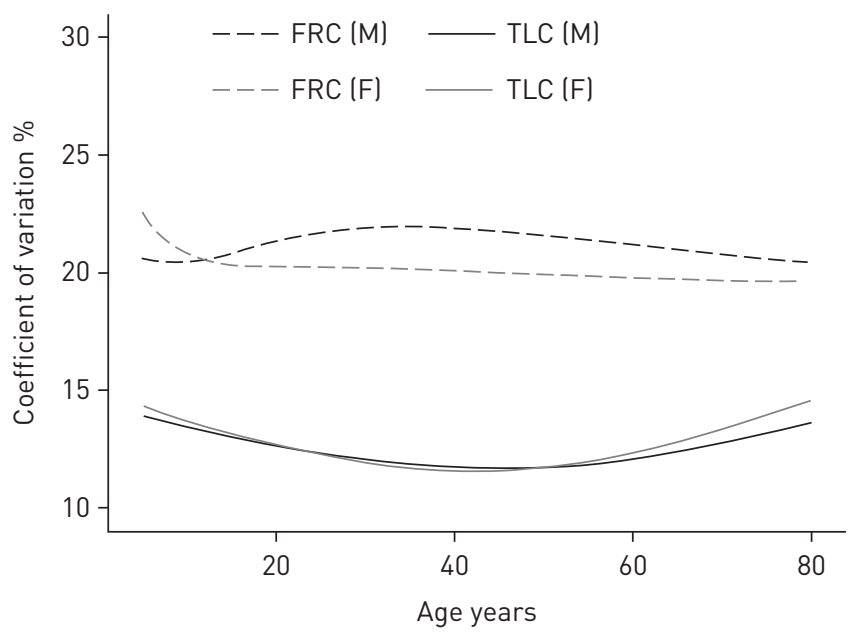

The predicted RV and RV/TLC equations are presented in table 3 and figure 2. Differences in RV values between the different techniques were greater than those observed for FRC and TLC (supplementary table S1). In addition, reference equations were created for ERV and IC (table 3, figure 2).

\section{Population variability}

The changes in the population variability (expressed as coefficient of variation) of FRC and TLC with age are presented in figure 3. The population variability of FRC was similar between males and females and relatively age-independent with an average coefficient of variation of $\sim 20 \%$. For TLC, younger children had more variable results than adolescents and adults, with variability between males and females being similar. Population variability of other indices tended to vary across the age range (supplementary figure S5) and was markedly increased for RV, and consequently RV/TLC in children and adults aged <40 years. In contrast, population variability was highest in older adults for ERV (supplementary figure S5). As a result, it is inappropriate to apply a fixed limit of normality for the majority of lung volume indices. The impact of age-related changes in RV and its influence on the upper limit of normal are highlighted in supplementary figure S6.

\section{Sensitivity analyses}

A significant proportion of the data submitted was obtained in either overweight (38.5\%) or obese (16.3\%) individuals. On average, the overweight and obese children and adults had lower FRC $\mathrm{z}$-scores $(-0.19 \pm 1.01$ for males, $-0.24 \pm 0.99$ for females) and lower ERV $z$-scores $(-0.19 \pm=1.0$ for males, $-0.25 \pm 0.99$ for females) (supplementary table S2). However, the majority of observations (95.1\%) were within $\pm 2 \mathrm{z}$-scores (supplementary figure S7). In the evaluation of the impact of BMI on a continuous scale, the weight bias was not observed for TLC or RV, but was observed for FRC and ERV (supplementary figure S7). On an individual level, the differences in observed values using the predicted values generated from the normal-weight population only (equations not provided), and including the overweight population are not dramatically different, with differences in predicted TLC, FRC and RV being $<75 \mathrm{~mL}$ and $<2.5 \%$ (supplementary table $\mathrm{S} 3$ ).

In addition, the influence of the largest dataset was evaluated. Firstly, we compared the overlap in observed values for the largest centre and all other centres (supplementary figure S8); visually there was no bias detected and the observations from all other centres overlapped with the largest centre. We tested equations developed using a smaller subset of the largest centre $(n=400)$; the predicted equations were similar if a smaller subset of the largest centre was used instead of the full sample size (data not shown).

\section{Comparison with existing reference values}

Overall, the GLI equations were similar to most previously published equations for FRC and TLC (figure 4), with closer agreement during childhood and adolescence than in adulthood. The biggest differences were observed for RV and RV/TLC in adults, particularly between the current GLI equations and the Goldman and Becklake [14] and the European Coal and Steel Community (ECSC) equations (figure 4, supplementary figure S9).

The VC equations were similar to those of FVC measured by spirometry (supplementary figure S10 and table S4). However, the VC equations are based on a significantly smaller sample size and limited data in early adulthood which could explain the differences. The differences in z-scores between predicted VC 
a)

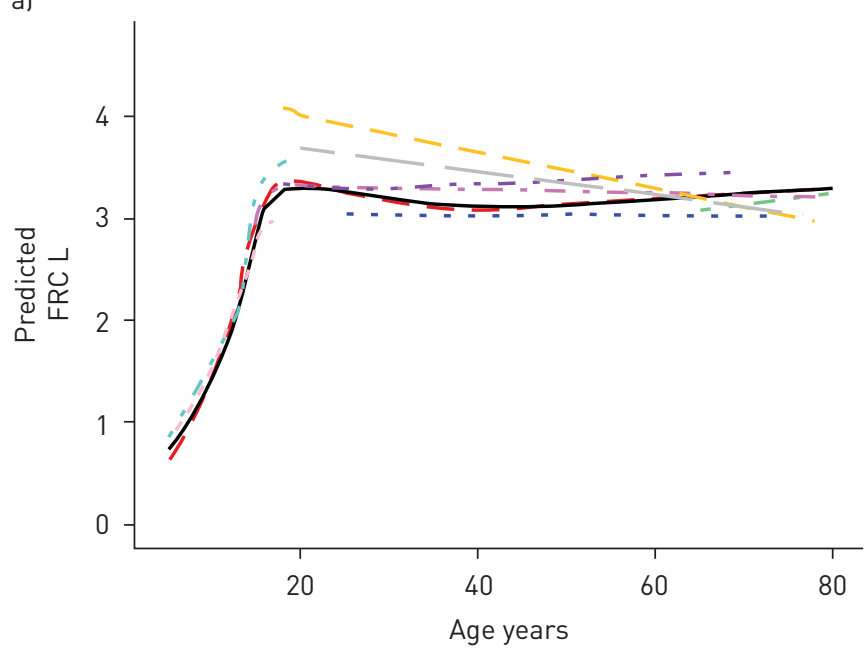

c)

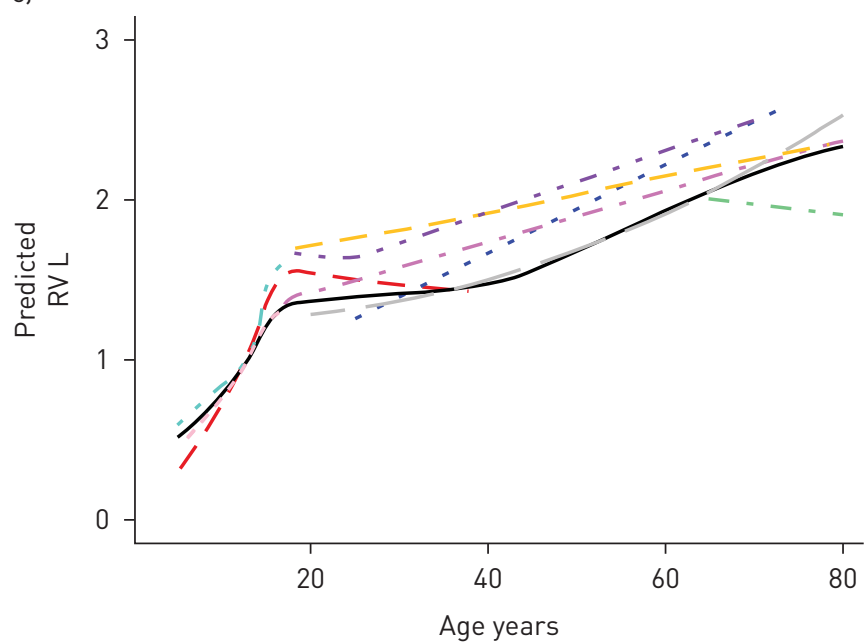

b)

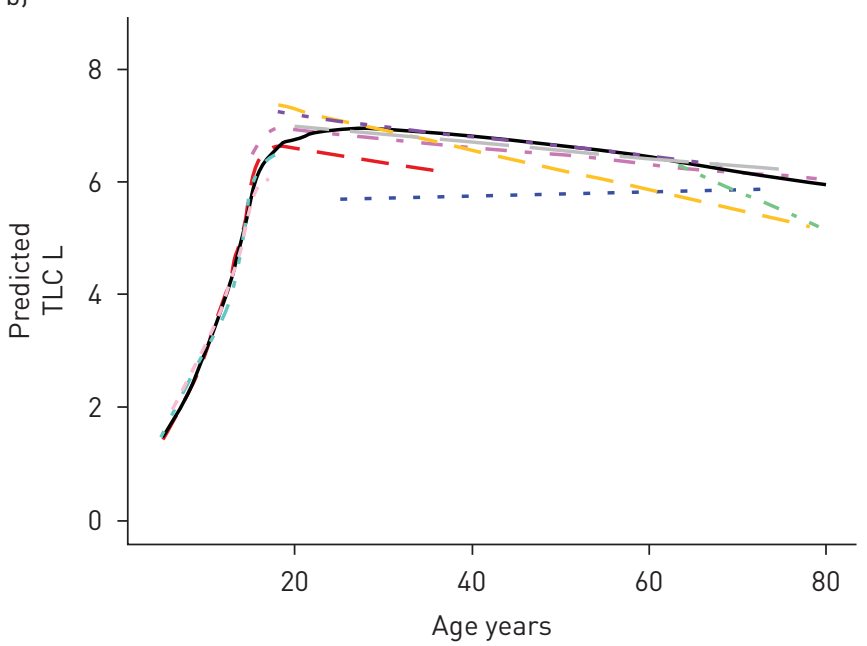

d)

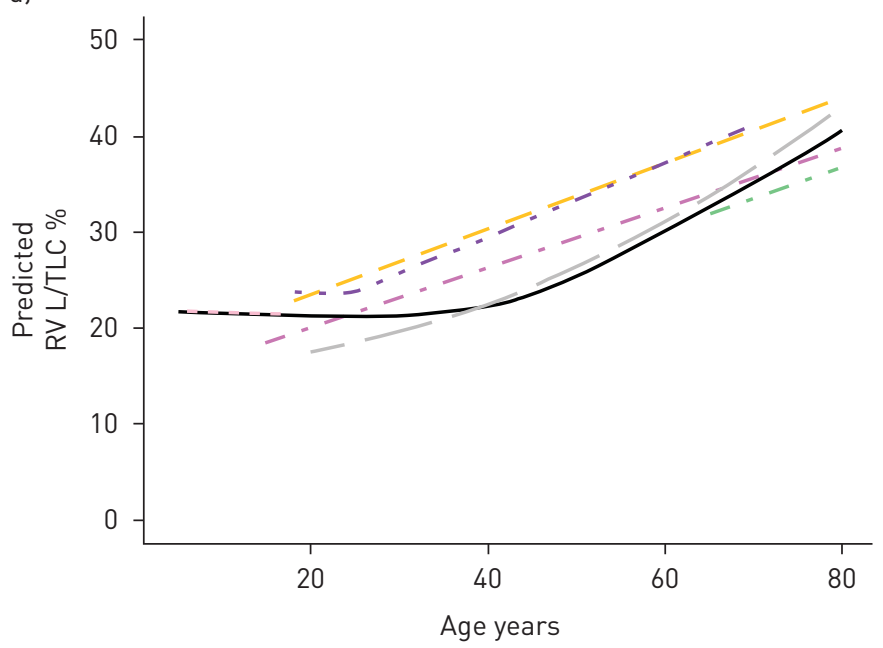

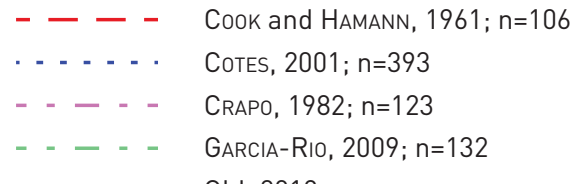

GLI, 2019$$
\text { - _ - - Goldman and BeckLAKE, 1959; n=44 }
$$$$
\text { ... - . . QuANJer (ECSC), 1993; n=NR }
$$$$
\cdots-\cdots \text { RosentHAL, 1993; } \mathrm{n}=772
$$$$
-\longrightarrow \text { Verbanck, 2016; } \mathrm{n}=128
$$$$
-\cdots-\cdots=\text { ZAPLETAL, 1977; n=NR }
$$

FIGURE 4 Comparison with commonly used reference equations in males. A comparison of commonly used equations in females are presented in supplementary figure S9. Predicted a) functional residual capacity (FRC), b) total lung capacity (TLC), c) residual volume (RV) and d) RV/TLC. GLI: Global Lung Function Initiative; ECSC: European Coal and Steel Community, NR: not reported.

(from the reference equations derived here) and FVC (derived from the GLI 2012 spirometry equations) were small (mean \pm SD $z$-score difference $0.19 \pm 0.89$ ).

\section{Physiologically relevant differences}

The GLI Network has previously reported a physiologically relevant difference in spirometry equated to $0.5 \mathrm{z}$-score. For the reported indices a $0.5 \mathrm{z}$-score difference at a population level equated to change of $\sim 10 \%$ for FRC and $\sim 8 \%$ for IC and was stable with age. For TLC the $0.5 \mathrm{z}$-scores equate to $\sim 8 \%$ in young children and older adults and $\sim 5 \%$ in adults aged 30-50 years. A 0.5 difference in $\mathrm{z}$-score was associated with larger differences in ERV and RV, starting at $\sim 13 \%$ in childhood and increasing to $~ 30 \%$ in older adults. As the population variability varies with age and sex, these are approximations.

\section{Discussion}

We report the collation and analysis of lung function indices derived from static lung volume measurements (body plethysmography and gas dilution techniques) obtained in healthy participants of 
European ancestry. In healthy individuals, lung volume data collected for two gas dilution techniques (nitrogen washout and helium dilution) and body plethysmography demonstrated remarkable overlap. The development of sex-specific reference equations that summarise the height and age-related changes in static lung volume indices across the age spectrum for individuals of European ancestry should improve the standardisation of the reporting and interpretation of lung volume measurements. The combination of data derived from multiple equipment types in healthy individuals will ensure broad generalisability across current clinical practice. The primary limitation of the presented lung volumes reference ranges is that these are limited to people of European ancestry. The GLI Network has implemented an active GLI lung function data repository, is actively promoting the submission of lung function data in individuals of non-European ancestry and will aim to address this gap in the future. It is not clear if these GLI lung volume equations are appropriate for persons of non-European ancestry. If GLI lung volume reference ranges are applied in individuals of non-European ancestry, this should be clearly stated to ensure results are not misinterpreted.

Like GLI reference ranges for spirometry [1] and transfer factor [2], sex-specific equations were derived with both age and height being independent and significant predictors for lung volume indices. Specifically, the relationship between age and height is multiplicative so that periods of rapid growth and development (e.g. puberty) are considered. Notably, the population variability (coefficient of variation) was $20 \%$ for FRC which equates to a range of normal of $60-140 \%$, much wider than the commonly used threshold of $80-120 \%$. The normal range is also much wider and age-dependent for the remainder of the lung volume indices (e.g. TLC, ERV and IC) meaning that the limits of normal are not a fixed value that can be applied across the entire age spectrum. The GLI lung volume reference ranges, and visual display relative to the normal range, should help facilitate interpretation of lung volume values relative to the normal range as compared with using a single fixed cut-off for all indices. Standardised equipment specifications and measurement protocols have helped to reduce the variability and biases between devices and centres [8]; nonetheless, the observed population variability is much greater than that within-subject repeatability and reproducibility of these tests [26].

It is noteworthy that FRC and TLC growth appears complete before 20 years, and then plateaus for males and females. This differs significantly from that which is observed in spirometry where the plateau occurs in the mid-20s, related to peaking of respiratory muscle strength [3]. One possible explanation for this is the limited data in this age range; however, there were sufficient data in childhood and later adulthood such that the smoothed equations based on a relatively smaller dataset in early adulthood should reflect the underlying age-related changes. In addition, the total number of observations between 20 and 40 years of age $(n=948)$ still represents the largest collection of normal data in this age group published to date.

The practice of reporting FVC and VC as separate indices varies between laboratories and there are no specific guidelines as to which should be reported. We report VC equations so that these can be summarised separately from FVC on reports, noting that the predicted VC will be generally higher than the predicted FVC from spirometry. Many laboratories use the predicted FVC values for both FVC and $\mathrm{VC}$, or clinicians frequently compare FVC and VC derived from separate predicted equations. Laboratories that choose to report different predicted values for FVC and VC, using GLI equations will be able to characterise physiological differences between measured values of FVC and VC, particularly in the presence of airway obstruction [27, 28]. While the GLI equations do not provide predicted values for $\mathrm{FEV}_{1} / \mathrm{VC}$, the availability of a predicted $\mathrm{VC}$ value will allow clinicians to compare two methods for defining obstruction, which may be useful for patients whose $\mathrm{FEV}_{1} / \mathrm{FVC}$ is close to the LLN. The Standardization of Spirometry 2019 update [29] provides specific guidelines for acceptability and repeatability of VC and IC and the GLI lung volumes reference ranges provide a significant advantage in reporting the predicted values for these two parameters.

We report that the $\mathrm{FEV}_{1}, \mathrm{FVC}$ and $\mathrm{FEV}_{1} / \mathrm{FVC}$ ratio of a subset of participants with both spirometry and lung volume measurements showed good agreement with the larger GLI spirometry cohort. A similar good fit was observed between the $T_{\text {LCO }}$ cohort and the larger GLI spirometry cohort [2]. Considered together, these data suggest that it will be possible to use the GLI spirometry, gas transfer and lung volume reference ranges in conjunction with each other. The consistency of analytical methods and approach should offer increased interpretation certainty when compared to the traditional approach of using lung function reference ranges derived from smaller populations, with different statistical models applied and developed by different investigators. In particular, the use of all-ages reference ranges eliminates errors associated with switching between separately developed paediatric and adult reference ranges.

\section{Technique and equipment differences}

Theoretical differences exist between the gas dilution and plethysmographic methods for measuring static lung volumes; however, the data gathered for the current study displayed significant overlap in healthy 
individuals. Multiple studies have demonstrated important differences between lung volume methods in patients who have airway obstruction [30-34], whereas in studies that measure healthy subjects, as in the GLI data, multiple-breath gas dilution and plethysmography produce small differences that are clinically insignificant. Furthermore, the most recent standardisation and equipment specification efforts have meant that the measurement accuracy of most devices is very good, and demonstrate minimal differences in model systems [35]. In this study the relative differences in FRC between plethysmography and helium and nitrogen gas dilution techniques were $<120 \mathrm{~mL}$ and within the technical accuracy of the measurement technique [8].

\section{Obesity}

The presented GLI lung volume equations include overweight and obese individuals. Obesity may influence both lung function and may be a risk factor for lung disease. Nonetheless, all individuals included in the dataset were nonsmokers without a history of respiratory disease. Furthermore, sensitivity analysis demonstrated minimal differences compared to equations derived for normal-weight subjects only. Reduced FRC (and ERV) and increased RV have been described in overweight and obese individuals [25]; however, the differences in predicted FRC between obese and nonobese groups in our cohort were small (supplementary table S4 has a worked example of differences in a representative male and female).

Exclusion of overweight and obese individuals would increase the predicted values slightly and would represent "ideal" health and allow assessment of the impact of weight on lung volumes. Conversely, excluding overweight and obese individuals from the derived equations excludes a significant proportion of individuals with no documented evidence of lung disease. These "ideal weight" equations would increase the risk of identifying "abnormalities" in lung function in obese individuals that may not be clinically relevant. If lung volumes are used to confirm restrictive disease, then the inclusion of obese individuals in the normative data is unlikely to impact interpretation. If the lung volume measurement is used to determine if there is air trapping, abdominal adiposity will affect FRC values such that they are reduced.

\section{Comparison with existing reference equations}

The 2005 ATS/ERS interpretation document [7] outlined 16 lung volume reference ranges, but did not make specific recommendations regarding which equations should be used for specific populations. Widely used equations, such as the ECSC, Goldman and BeCKLAKe [14] and others are limited because they are only available for adults $[15,17]$. The currently available paediatric reference equations do not adequately deal with changes during puberty or the continuum between paediatric and adult equations, resulting in discontinuous breaks at arbitrary points [16]. Overall, the GLI equations for FRC and TLC aligned well with previously published equations, especially for children. The biggest differences were observed for RV and RV/TLC equations. The observed differences are likely to influence clinical practice, as individuals previously identified as normal or "abnormal" may have a different interpretation using the GLI lung volume equations. Recalculation of historical measurements will be necessary to avoid misinterpretation of changes in an individual patient's predicted lung function.

\section{Implementation}

The format of the GLI lung volume reference ranges are identical to the GLI spirometry and gas transfer equations, which will facilitate implementation into many devices which already have the GLI lung function equations programmed. The prediction equations (table 3) and look-up tables and an online calculator (www.lungfunction.org) are provided, and a worked example is included in the supplementary material. Health professionals need to carefully consider the impact of changing their current reference ranges on patient care [36]. Changing from currently utilised reference ranges (for example ECSC) to the GLI reference range will probably lead to differences in the proportion of patients classified as having abnormal lung function. Studies of this issue have been reported for spirometry [37, 38] and the single-breath gas transfer test [39-41] and all have broadly noted that the impact of changing to GLI equations for these tests varies with age and sex and can impact on the potential eligibility for treatments [42]. Studies assessing the impact of switching to the GLI lung volume equations will be required to help inform health professionals and their patients.

\section{Limitations}

The derived GLI lung volume reference equations are from participants of European ancestry and may not be appropriate for individuals of non-European ancestry. The data available for collation by the GLI lung volumes task force is not inclusive of all published lung volume data in healthy participants. The task force contacted corresponding authors of all published studies and advertised the opportunity to submit data through international and national respiratory societies. However, some centres declined or were unable to gain appropriate approvals to submit their published data. The increased move to open access to 
data collected as part of publicly funded research should reduce the impact of this issue in the future. In this study a single centre contributed nearly half of the data. While this represents a large relative contribution, both the spirometry and $T_{\mathrm{LCO}}$ datasets comprised many small datasets and one or two large datasets. In all instances the range of data observed in the smaller datasets were contained within the range of the large dataset. Although this could represent a bias towards a single equipment or centre, the data do not support this (supplementary figure S8). There is the potential for this inherent bias to impact the derived reference equations, especially when systematic equipment or protocol differences may influence results. Ideally, multiple large datasets would be available; however, the current GLI lung volumes equations, although imperfect, represent a significant step towards standardised reporting and interpretation. There were limited data for RV in children and young adults. Until more data are available, we recommend the equations for RV and RV/TLC should be applied with caution in children and young adults. The GLI data repository is now open and accepting additional datasets, with the aim to routinely validate and update existing equations as needed.

\section{Conclusions}

The ERS GLI reference values for lung volumes provide all-ages generalisable reference to standardise the reporting and interpretation of lung volumes measurements in European ancestry populations. Data from non-European ancestries are urgently required to allow the expansion of these equations for the global population.

Acknowledgements: The authors offer their sincere thanks to all the staff and participants at each of the participating centres for their contributions to the collection of the original data and for generously sharing their data with the GLI Network, and to Kim Carter of Telethon Kids Institute and Data Analysis Australia (Perth, Australia) for his assistance in developing and managing the data repository platform. We would like to thank Celine Genton and her team from the European Respiratory Society for their support of the GLI Network.

The contributing GLI Network members (in alphabetical order) include: Bert Arets, University Medical Centre, Utrecht, the Netherlands; Helmi Ben Saad, Faculty of Medicine of Sousse, Sousse, Tunisia; Simona Costanzo, IRCCS Neuromed, Pozzilli, Italy; Rachel Foong, Telethon Kids Institute, Perth, Australia; Monika Gappa, Marienhospital, Wesel, Germany; Francisco Garcia-Rio, Hospital Universitario La Paz, Madrid, Spain; Laura Gochicoa-Rangel, Instituto Nacional de Enfermedades Respiratorias, Mexico City, Mexico; Licia Iacoviello, IRCCS Neuromed, Pozzilli, Italy, and University of Insubria, Varese, Italy; Philipp Latzin, Bern University Hospital, Berne, Switzerland; Pablo Ferrer Lopez, Papeete, French Polynesia; Sooky Lum, Institute of Child Health, London, UK; Fabrice Rannou, Centre hospitalier universitaire de Brest, Brest, France; Shannon Simpson, Telethon Kids Institute, Perth, Australia; Maureen Verheggen, Perth Children's Hospital, Perth, Australia; Debbie Zagami, Gold Coast Hospital and Health Service Southport, Australia; and Gerald Zavorsky, University of California - Davis Medical Center, USA. The GLI Executive (in alphabetical order) include: Brendan Cooper, Queen Elizabeth Hospital, Birmingham, UK; Carlos Fragoso, Yale School of Medicine, New Haven, USA; Laura Gochicoa Rangel, Instituto Nacional de Enfermedades Respiratorias, Mexico City, Mexico; Graham Hall, Telethon Kids Institute and Curtin University, Perth, Australia; Alexander John Henderson, University of Bristol, Bristol, UK; Thi Tuyet Lan Le, University Medical Center, Hồ Chí Minh City, Vietnam; Refiloe Masekela, University of KwaZulu-Natal, Durban, South Africa; Gail McAvay, Yale School of Medicine, New Haven, USA; Meredith McCormack, Harvard Medical School, Boston, USA; Pippa Powell, European Lung Foundation, Sheffield, UK; Sanja Stanojevic, Hospital for Sick Children, Toronto, Canada; and Irene Steenbruggen, Isala Klinieken, Zwolle, the Netherlands.

Conflict of interest: G.L. Hall reports grants from European Respiratory Society, during the conduct of the study; other (equipment loan) from Ndd, outside the submitted work. N. Filipow has nothing to disclose. G. Ruppel reports personal fees for lectures from MGC Diagnostics, outside the submitted work. T. Okitika has nothing to disclose. B. Thompson reports grants from European Respiratory Society, during the conduct of the study. J. Kirkby has nothing to disclose. I. Steenbruggen has nothing to disclose. B.G. Cooper has nothing to disclose. S. Stanojevic reports other (task force contribution to the statistical analyses) from ERS, during the conduct of the study.

Support statement: The ERS GLI lung volume task force was funded by the European Respiratory Society with in-kind support from Telethon Kids Institute and Curtin University in Perth, Australia and the Sick Kids Research Institute in Toronto, Canada. Funding information for this article has been deposited with the Crossref Funder Registry.

\section{References}

1 Quanjer PH, Stanojevic S, Cole TJ, et al. Multi-ethnic reference values for spirometry for the 3-95-yr age range: the global lung function 2012 equations. Eur Respir J 2012; 40: 1324-1343.

2 Stanojevic S, Graham BL, Cooper BG, et al. Official ERS technical standards: Global Lung Function Initiative reference values for the carbon monoxide transfer factor for Caucasians. Eur Respir J 2017; 50: 170010.

3 Quanjer $\mathrm{PH}$, Stanojevic S, Stocks J, et al. Changes in the $\mathrm{FEV}_{1} / \mathrm{FVC}$ ratio during childhood and adolescence: an intercontinental study. Eur Respir J 2010; 36: 1391-1399.

4 Hall GL, Stanojevic S, GLI Network Executive. The Global Lung Function Initiative (GLI) Network ERS Clinical Research Collaboration: how international collaboration can shape clinical practice. Eur Respir J 2019; 53: 1802277.

5 Stanojevic S, Stocks J, Bountziouka V, et al. The impact of switching to the new global lung function initiative equations on spirometry results in the UK CF registry. J Cyst Fibros 2014; 13: 319-327.

6 Wapenaar M, Miedema JR, Lammering CJ, et al. The impact of the new Global Lung Function Initiative $T_{\mathrm{LCO}}$ reference values on trial inclusion for patients with idiopathic pulmonary fibrosis. Eur Respir J 2019; 53: 1801895. 
7 Pellegrino R, Viegi G, Brusasco V, et al. Interpretative strategies for lung function tests. Eur Respir J 2005; 26: 948-968.

8 Wanger J, Clausen JL, Coates A, et al. Standardisation of the measurement of lung volumes. Eur Respir J 2005; 26 511-522.

9 Cook CD, Hamann JF. Relation of lung volumes to height in healthy persons between the ages of 5 and 38 years. J Pediatr 1961; 59: 710-714.

10 Cordero PJ, Morales P, Benlloch E, et al. Static lung volumes: reference values from a Latin population of Spanish descent. Respiration 1999; 66: 242-250.

11 Cotes JE, Chinn DJ, Reed JW. Body mass, fat percentage, and fat free mass as reference variables for lung function: effects on terms for age and sex. Thorax 2001; 56: 839-844.

12 Crapo RO, Morris AH, Gardner RM. Reference values for pulmonary tissue volume, membrane diffusing capacity, and pulmonary capillary blood volume. Bull Eur Physiopathol Respir 1982; 18: 893-899.

13 Garcia-Rio F, Dorgham A, Pino JM, et al. Lung volume reference values for women and men 65 to 85 years of age. Am J Respir Crit Care Med 2009; 180: 1083-1091.

14 Goldman HI, Becklake MR. Respiratory function tests; normal values at median altitudes and the prediction of normal results. Am Rev Tuberc 1959; 79: 457-467.

15 Quanjer PH, Tammeling GJ, Cotes JE, et al. Lung volumes and forced ventilatory flows. Eur Respir J 1993; 6: Suppl 16, 5-40.

16 Rosenthal M, Cramer D, Bain SH, et al. Lung function in white children aged 4 to 19 years: II - single breath analysis and plethysmography. Thorax 1993; 48: 803-808.

17 Zapletal A, Paul T, Samánek M. [Significance of contemporary methods of lung function testing for the detection of airway obstruction in children and adolescents (author's transl)]. Z Erkr Atmungsorgane 1977; 149: 343-371.

18 Verbanck S, Van Muylem A, Schuermans D, et al. Transfer factor, lung volumes, resistance and ventilation distribution in healthy adults. Eur Respir J 2016; 47: 166-176.

19 Carter KW, Francis RW, Carter KW, et al. ViPAR: a software platform for the Virtual Pooling and Analysis of Research Data. Int J Epidemiol 2016; 45: 408-416.

20 Rigby RA, Stasinopoulos DM. Smooth centile curves for skew and kurtotic data modelled using the Box-Cox power exponential distribution. Stat Med 2004; 23: 3053-3076.

21 Cole TJ, Stanojevic S, Stocks J, et al. Age- and size-related reference ranges: a case study of spirometry through childhood and adulthood. Stat Med 2009; 28: 880-898.

22 Stanojevic S, Wade A, Stocks J, et al. Reference ranges for spirometry across all ages: a new approach. Am J Respir Crit Care Med 2008; 177: 253-260.

23 Cole TJ, Green PJ. Smoothing reference centile curves: the LMS method and penalized likelihood. Stat Med 1992; 11: $1305-1319$.

24 Kuczmarski RJ, Ogden CL, Grummer-Strawn LM, et al. CDC growth charts: United States. Adv Data 2000; 314: $1-27$.

25 Jones RL, Nzekwu MM. The effects of body mass index on lung volumes. Chest 2006; 130: 827-833.

26 Hankinson JL, Stocks J, Peslin R. Reproducibility of lung volume measurements. Eur Respir J 1998; 11: 787-790.

27 Yuan W, He X, Xu QF, et al. Increased difference between slow and forced vital capacity is associated with reduced exercise tolerance in COPD patients. BMC Pulm Med 2014; 14: 16.

28 Brusasco V, Pellegrino R, Rodarte JR. Vital capacities in acute and chronic airway obstruction: dependence on flow and volume histories. Eur Respir J 1997; 10: 1316-1320.

29 Graham BL, Steenbruggen I, Miller MR, et al. Standardization of Spirometry 2019 update. An official American Thoracic Society and European Respiratory Society technical statement. Am J Respir Crit Care Med 2019; 200: e70-e88.

30 Milite F, Lederer DJ, Weingarten JA, et al. Quantification of single-breath underestimation of lung volume in emphysema. Respir Physiol Neurobiol 2009; 165: 215-220.

31 Pesola GR, Magari RT, Dartey-Hayford S, et al. Total lung capacity: single-breath methane dilution versus plethysmography in normals. Respirology 2007; 12: 291-294.

32 Punjabi NM, Shade D, Wise RA. Correction of single-breath helium lung volumes in patients with airflow obstruction. Chest 1998; 114: 907-918.

33 Tantucci C, Bottone D, Borghesi A, et al. Methods for measuring lung volumes: is there a better one? Respiration 2016; 91: 273-280

34 Kane $\mathrm{M}$, Rayment $\mathrm{JH}$, Jensen $\mathrm{R}$, et al. Correcting for tissue nitrogen excretion in multiple breath washout measurements. PLoS One 2017; 12: e0185553.

35 Jensen RL, Teeter JG, England RD, et al. Instrument accuracy and reproducibility in measurements of pulmonary function. Chest 2007; 132: 388-395.

36 Derom E, Liistro G, Oostveen E, et al. Launching Global Lung Function Initiative reference values in Belgium: tips and tricks. Eur Respir J 2018; 52: 1800922.

37 Brazzale DJ, Hall GL, Pretto JJ. Effects of adopting the new Global Lung Function Initiative 2012 reference equations on the interpretation of spirometry. Respiration 2013; 86: 183-189.

38 Quanjer PH, Brazzale DJ, Boros PW, et al. Implications of adopting the Global Lungs Initiative 2012 all-age reference equations for spirometry. Eur Respir J 2013; 42: 1046-1054.

39 Brazzale DJ, Seccombe LM, Welsh L, et al. Effects of adopting the Global Lung Function Initiative 2017 reference equations on the interpretation of carbon monoxide transfer factor. Eur Respir J 2020; 55: 1901905.

40 Oostveen E, Derom E, Vints AM, et al. Comparison of ERS'93 to the newly published GLI'17 reference values for carbon monoxide transfer factor. Respir Med 2019; 146: 113-115.

41 Soumagne $\mathrm{T}$, Guillien A, Chambellan A, et al. Global Lung Initiative equations for pulmonary hypertension screening in systemic sclerosis. Eur Respir J 2018; 52: 1800528.

42 Burgess A, Goon K, Brannan JD, et al. Eligibility for anti-fibrotic treatment in idiopathic pulmonary fibrosis depends on the predictive equation used for pulmonary function testing. Respirology 2019; 24: 988-995. 